\title{
Calidad de vida y educación en tiempos de pandemia: Una reflexión desde las Ciencias del Movimiento Humano
}

\section{Quality of Life and Education in Time of Pandemic: A Reflection From the Human Movement Sciences}

\section{Qualidade de vida e educação em tempos de pandemia: Uma reflexão das Ciências do Movimento Humano}

María Amalia Penabad-Camacho

Universidad Nacional

ROR: https://ror.org/01t466c14

Escuela Ciencias del Movimiento Humano y Calidad de Vida

Heredia, Costa Rica

maria.penabad.camacho@una.ac.cr

(iD) https://orcid.org/0000-0002-5095-5775

Magally Márquez-Barquero

Universidad Nacional

ROR: https://ror.org/01t466c14

Escuela Ciencias del Movimiento Humano y Calidad de Vida

Heredia, Costa Rica

magally.marquez.barquero@una.ac.cr

https://orcid.org/0000-0002-1498-4798

Lady Peña-Conejo

Universidad Nacional

ROR: https://ror.org/01t466c14

Escuela Ciencias del Movimiento Humano y Calidad de Vida

Heredia, Costa Rica

lady.pena.conejo@una.ac.cr

https://orcid.org/0000-0003-1789-5499

Inés Revuelta-Sánchez

Universidad Nacional

ROR: https://ror.org/01t466c14

Escuela Ciencias del Movimiento Humano y Calidad de Vida

Heredia, Costa Rica

ines.revuelta.sanchez@una.ac.cr

https://orcid.org/0000-0003-4560-1953 
https://doi.org/10.15359/ree.24-S.13

https://www.revistas.una.ac.cr/index.php/educare

educare@una.ac.cr

Resumen: En el contexto educativo, la Universidad Nacional determinó procedimientos para enfrentar el confinamiento por el COVID-19. Esto representó un nuevo estilo de vida para la comunidad universitaria y provocó afectaciones en su salud y calidad de vida. Partiendo desde el movimiento humano, el siguiente editorial hace un llamado de atención y ofrece recomendaciones para construir acciones que permitan contrarrestar los efectos negativos de la pandemia y aprovechar las oportunidades emergentes.

Palabras claves: COVID-19; salud; confinamiento.

Abstract: In the educational context, the Universidad Nacional determined procedures to face social confinement by COVID-19. This represented a new lifestyle for the university community and affected their health and quality of life. From the human movement standpoint, the following editorial calls for atention and offers recommendations to build actions in order to counteract the negative effects of the pandemic and take advantage of emerging oportunities.

Keywords: COVID-19; health; lockdown.

Resumo: No âmbito educacional, a Universidade Nacional determinou procedimentos para enfrentar o confinamento pelo COVID-19. Isso representou um novo estilo de vida para a comunidade universitária e afetou sua saúde e qualidade de vida. Partindo do movimento humano, o editorial a seguir chama a atenção e oferece recomendações para construir ações para neutralizar os efeitos negativos da pandemia e aproveitar as oportunidades emergentes.

Palavras-chaves: COVID-19; saúde; confinamento.

Recibido: 25/08/2020 Aceptado: 02/09/2020

\section{Introducción}

A raíz de la propagación del COVID-19, Costa Rica ha enfrentado una transformación en las áreas de la salud, la economía y lo social. En el contexto educativo, la Universidad Nacional determinó procedimientos para enfrentar el confinamiento, ampliando la modalidad de teletrabajo e implementando la presencialidad remota para continuar con el ciclo lectivo. Se destacó el esfuerzo institucional por brindar los recursos tecnológicos y la capacitación para lograr el acceso igualitario de la comunidad universitaria, lo que posibilitó mantener los procesos. A pesar de haber dado continuidad al quehacer sustantivo institucional, en forma paralela, se produjeron afectaciones para quienes experimentaron cambios en su calidad de vida.

Los trabajos de Mera-Mamián, et al. (2020); Moreno, et al. (2020) y Reimers y Schleicher (2020), señalan el impacto provocado por la pandemia en las dimensiones que integran la calidad de vida, así como alteraciones en la salud. En este sentido, se hace un llamado de atención para construir 
https://doi.org/10.15359/ree.24-S.13 https://www.revistas.una.ac.cr/index.php/educare educare@una.ac.cr

acciones que conduzcan a contrarrestar las afectaciones negativas de la situación y aprovechar las oportunidades emergentes. El propósito de este editorial es brindar recomendaciones desde las ciencias del movimiento humano (MH) y calidad de vida (CV) para motivar la implementación de prácticas saludables durante el confinamiento en el contexto educativo.

La construcción de prácticas saludables a partir del distanciamiento social, obliga a asumir de manera asertiva, un enfoque integral en el cual las Ciencias del $\mathrm{MH}$ se convierten en un medio para mantenerse en movimiento a nivel físico, intelectual, espiritual, ocupacional, psicoemocional y con particular importancia en lo social. Cuando comprendemos que el movimiento, tanto a lo interno como a lo externo del cuerpo, es un principio de vida, se posibilita estar en constante desplazamiento en lo corporal, espacial, emocional y mental; algo muy necesario en tiempos de pandemia y más allá de ella.

Desde lo social, "se hace necesario develar potenciales estrategias de AF [actividad física] y hábitos saludables que puedan ser adoptadas desde el confinamiento en casa por el núcleo familiar" (Mera-Mamián et al., 2020, p.167). Esto implica considerar no solo las formas tradicionales del $\mathrm{MH}$, sino también el planteamiento desde lo sencillo a lo complejo, con relación a las posibles maneras y medios para mantenerse en movimiento desde un comportamiento basado en la toma de conciencia y responsabilidad que corresponde asumir, en primera instancia a nivel personal por medio del autocuidado (Moreno et al., 2020), para luego unir esfuerzos en el entorno inmediato de convivencia y se propone producir encadenamientos de movimiento en procura de una mejor $\mathrm{CV}$ desde las ciencias del $\mathrm{MH}$.

La medida del confinamiento aumenta las conductas sedentarias, por lo que se recomienda incorporar $\mathrm{MH}$ dentro de la casa, de bajo costo y fácil acceso que ayude a prevenir el desacondicionamiento físico, un síndrome que aparece en las personas que hacen reposo prolongado y se relaciona con la pérdida de masa muscular, de flexibilidad y de fuerza. La actividad que se elija ha de promover la salud y el disfrute, tanto de manera individual como en la compañía de las personas con las que se asume el aislamiento y que esto, se adopte como un estilo de vida (Mera-Mamián et al., 2020).

Al desarrollar las actividades académicas y laborales desde la casa, es útil realizar pausas activas de tres a cinco minutos por hora, ponerse de pie y caminar, para movilizar las piernas y favorecer la circulación sanguínea. Además, en el caso de las personas mayores de 18 años, acumular al menos 150 minutos de MH por semana, con actividades a intensidad moderada. Se debe realizar un calentamiento con estiramientos dinámicos y movilidad articular; incluir rutinas de ejercicios que involucren los grandes grupos musculares al menos dos días por semana. El MH no debe generar molestias y si no se tolera, cambiarlo por otro que se adecúe a las posibilidades individuales. También, participar de las actividades domésticas, incluir técnicas de respiración 
https://doi.org/10.15359/ree.24-S.13

https://www.revistas.una.ac.cr/index.php/educare

educare@una.ac.cr

consciente y meditación, porque más minutos de $\mathrm{MH}$ se asocian con beneficios en el manejo de la ansiedad, los ataques de pánico, el estrés y el insomnio ligados con el confinamiento.

En el ámbito educativo a lo interno de la UNA, se deben propiciar más acciones en relación con el $\mathrm{MH}$ destinadas a combatir la deserción estudiantil, mantener la calidad académica y promover la salud de la comunidad universitaria. El MH debería estar presente desdela mediación pedagógica en el desarrollo de los cursos de cada campo disciplinar. Reimers y Schleider (2020), señalan que una pandemia es un desafío adaptativo por excelencia y a partir del $\mathrm{MH}$, se pueden mitigar las consecuencias asociadas a ella. Para una mayor promoción del $\mathrm{MH}$ se propone la creación de una Comisión de Gestión de la Calidad de Vida, que integre facultades, centros y sedes con el fin de observar, monitorear y vigilar la CV de la institución y sus personas.

La pandemia ha presentado una oportunidad para que las personas asuman una nueva responsabilidad a nivel individual y colectivo sobre su CV. El ámbito de la educación es instrumental para promover el conocimiento con un enfoque democrático que no priorice un grupo sobre otro, basado en un tratamiento eficaz en la gestión que hacen las personas de su vida. Es un momento histórico que evidencia la importancia del $\mathrm{MH}$; en el ejercicio y disfrute del derecho básico de las personas a la salud y una vida con calidad.

Nota general: Corrección de estilo y traducciones a cargo de las autoras.

\section{Referencias}

Mera-Mamián, A. Y., Tabares-González, E., Montoya-González, S., Muñoz-Rodríguez, D. I. y Monsalve-Vélez, F.(2020). Recomendaciones prácticas para evitar el desacondicionamiento físico durante el confinamiento por pandemia asociada a COVID-19. Universidad y Salud, 22(2), 166-177. https://doi.org/10.22267/rus.202202.188

Moreno, C., Wykes, T., Galderisi, S., Nordentoft, M., Crossley, N., Jones, N., Cannon, M., Correl, C., Byrne, L., Carr, S., Chen, E. Y., Gorwood, P., Johnson, S., Käkkäinen, H., Krystal, J., Lee, J., Lieberman, J., López-Jaramillo, C., Männikkö, M., ... Arango, C. (July, 2020). How mental health care should change as a consequence of the COVID-19 pandemic. The Lancet Psychiatry, 7(9), 813-824. https://doi.org/10.1016/S2215-0366(20)30307-2

Reimers, F. y Schleider, A. (2020). Un marco para guiar una respuesta educativa a la pandemia del 2020 del COVID-19. OECD. https://www.oei.es/Ciencia/Noticia/la-oei-difunde-un-informede-la-universidad-de-harvard 